\title{
BMJ Open Cost of hospitalised patients due to complicated urinary tract infections: a retrospective observational study in countries with high prevalence of multidrug-resistant Gram-negative bacteria: the COMBACTE-MAGNET, RESCUING study
}

\begin{abstract}
Laura Vallejo-Torres, ${ }^{1,2}$ Miquel Pujol, ${ }^{3,4}$ Evelyn Shaw, ${ }^{3,4}$ Irith Wiegand, ${ }^{5}$ Joan Miquel Vigo, ${ }^{6}$ Margaret Stoddart, ${ }^{7}$ Sally Grier, ${ }^{7}$ Julie Gibbs, ${ }^{7}$ Christiane Vank, ${ }^{5}$ Nienke Cuperus, ${ }^{8}$ Leo van den Heuvel, ${ }^{8}$ Noa Eliakim-Raz, ${ }^{9,10}$ Jordi Carratala, $, 3,4$ Cuong Vuong, ${ }^{5}$ Alasdair MacGowan, ${ }^{7}$ Tanya Babich, ${ }^{9,10}$ Leonard Leibovici,,${ }^{9,10}$ Ibironke Addy, ${ }^{5}$ Stephen Morris, ${ }^{1}$ on behalf of RESCUING Study Group and Study Sites
\end{abstract}

To cite: Vallejo-Torres L, Pujol M, Shaw E, et al. Cost of hospitalised patients due to complicated urinary tract infections: a retrospective observational study in countries with high prevalence of multidrug-resistant Gram-negative bacteria: the COMBACTE-MAGNET, RESCUING study. BMJ Open 2018;8:e020251. doi:10.1136/ bmjopen-2017-020251

- Prepublication history and additional material for this paper are available online. To view these files, please visit the journal online(http://dx.doi. org/10.1136/bmjopen-2017020251).

Received 25 0ctober 2017 Revised 7 December 2017 Accepted 16 January 2018

Check for updates

For numbered affiliations see end of article.

Correspondence to Dr Laura Vallejo-Torres; laura.vallejotorres@sescs.es

\section{ABSTRACT}

Objective Complicated urinary tract infections (cUTIs) impose a high burden on healthcare systems and are a frequent cause of hospitalisation. The aims of this paper are to estimate the cost per episode of patients hospitalised due to CUTI and to explore the factors associated with cUTI-related healthcare costs in eight countries with high prevalence of multidrug resistance (MDR).

Design This is a multinational observational, retrospective study. The mean cost per episode was computed by multiplying the volume of healthcare use for each patient by the unit cost of each item of care and summing across all components. Costs were measured from the hospital perspective. Patient-level regression analyses were used to identify the factors explaining variation in CUTI-related costs.

Setting The study was conducted in 20 hospitals in eight countries with high prevalence of multidrug resistant Gram-negative bacteria (Bulgaria, Greece, Hungary, Israel, Italy, Romania, Spain and Turkey).

Participants Data were obtained from 644 episodes of patients hospitalised due to cUTI.

Results The mean cost per case was $€ 5700$, with considerable variation between countries (largest value $€ 7740$ in Turkey; lowest value $€ 4028$ in Israel), mainly due to differences in length of hospital stay. Factors associated with higher costs per patient were: type of admission, infection source, infection severity, the Charlson comorbidity index and presence of MDR.

Conclusions The mean cost per hospitalised case of CUTI was substantial and varied significantly between countries. A better knowledge of the reasons for variations in length of stays could facilitate a better standardised quality of

\section{Strengths and limitations of this study}

- This is the first study to examine costs of hospitalised patients due to complicated urinary tract infection (CUTI) from a multinational point of view.

- It is focused on countries with a high prevalence of multidrug resistance bacteria where cUTI impose a significant burden.

- The study estimates the mean cost per case from a bottom-up perspective, which provided a high level of granularity and the basis for the assessment of sources of variation and drivers of healthcare costs.

- The design of the study did not include a control group to assess the extra length of stay and excess costs of patients who are admitted to hospital due to a different condition and develop urinary tract infection during their hospitalisation.

- Country-specific unit cost data were not appropriate for most countries, and therefore, we applied the same set of unit costs, as estimated in one country, Spain, to the rest of the countries.

care for patients with cUTI and allow a more efficient allocation of healthcare resources. Urgent admissions, infections due to an indwelling urinary catheterisation, resulting in septic shock or severe sepsis, in patients with comorbidities and presenting MDR were related to a higher cost.

\section{INTRODUCTION}

Urinary tract infections (UTIs) are highly prevalent worldwide. UTIs that occur in a 
normal genitourinary tract with no prior instrumentation are considered uncomplicated, whereas complicated UTIs (cUTIs) are associated with structural or functional abnormalities of the genitourinary tract or an underlying disease that interferes with host defence. ${ }^{1}$ cUTIs are a frequent cause of hospitalisation as well as a common complication during hospitalisation and have shown a higher prevalence of antimicrobial resistance compared with uncomplicated UTI. ${ }^{2}$ Due to the rapid emergence and dissemination of resistance to antimicrobial agents, leading in some cases to multidrug resistance (MDR), some patients with cUTI are left with few therapeutic options and may progress to more serious stages of the disease. $^{3}$

Currently, information about the burden of cUTI is scarce. Reports from the USA show that in the year 2000 cUTI accounted for more than 100000 hospital admissions, often as a result of pyelonephritis. ${ }^{4}$ Data from Europe are very limited, although the last point prevalence survey of European acute care hospitals estimated the prevalence of healthcare-associated infections to be $6 \%$; of these, UTI was the third most common infection $(19 \%) .{ }^{5}$ Based on these point prevalence data, the annual health burden of hospitalised patients with UTI was estimated to be 81.2 disability-adjusted life years per 100000 individuals in the general population. ${ }^{6}$

Despite this high burden to healthcare systems and the increased pressure for cost containment in healthcare, few studies have examined the costs of cUTIs. Some papers have measured the cost of community-acquired UTIs ${ }^{7-10}$ and nosocomial UTIs, ${ }^{11}{ }^{12}$ or both. ${ }^{13}$ Most of these studies were conducted in the USA, ${ }^{7811-13}$ while studies undertaken in European countries have mainly focused on women visiting primary care settings with suspected UTIs. ${ }^{9}{ }^{10}$ Some papers have estimated the impact of extended-spectrum beta-lactamase (ESBL)-producing Escherichia coli on the cost of UTI episodes requiring hospitalisation. ${ }^{1415}$ Estimating the magnitude of the financial impact of this prevalent and potentially avoidable condition is particularly useful for measuring the potential cost savings from averting a case, thereby emphasising the importance of prevention and the sizeable economic consequences of MDR. In addition, cost estimates might inform cost-effectiveness analyses that require data on episode costs in order to compare alternative courses of treatment related to this condition. Therefore, there is a need for data on the economic burden imposed to healthcare systems due to hospitalised cUTI patients, especially in countries with high prevalence of MDR.

In this paper, we present an analysis of the economic burden of cUTI in seven European countries plus Israel, all of which have a high prevalence of MDR. The aims of this study are to estimate the cost per case of hospitalised patients due to cUTI and to investigate the factors associated with cUTI-related healthcare costs.

The analyses reported in this paper are part of a larger project, 'REtrospective observational Study to assess the clinical management and outcomes of hospitalised patients with Complicated Urinary tract INfection in countries with high prevalence of multidrug resistant Gram-negative bacteria (RESCUING study)', with an overall aim of providing information about the epidemiology, clinical management, outcomes and healthcare costs of patients hospitalised with cUTI.

\section{MATERIALS AND METHODS \\ Setting}

This is a multinational observational, retrospective study conducted in 20 hospitals in eight countries (Bulgaria, Greece, Hungary, Israel, Italy, Romania, Spain and Turkey). Data were collected on patients who had a diagnosis of cUTI as the primary cause of hospitalisation and patients hospitalised for another reason but who developed cUTI during their hospitalisation from January 2013 to December 2014, based on International Classification of Diseases, 9th Revision (ICD-9) and ICD, 10th Revision (ICD-10) codes (ICD-9 Clinical Modification (CM) codes: 590.1, 590.10, 590.11, 590.2, 590.8, 590.80, 590.9, 595.0, 595.89, 595.9 and 599.0; ICD-10 CM codes: N10, N12, N13.6, N15.1, N15.9, N30.0, N30.8, N30.9 and N39.0). The study protocol has been published elsewhere. $^{16}$

In order to avoid selection bias, all consecutive patients who had ICD-9 or ICD-10 CM codes were reviewed at each site. All patients who met the inclusion criteria were selected for data collection. Inclusion criteria were patients with UTI and at least one of the following: indwelling urinary catheter, urinary retention, neurogenic bladder, obstructive uropathy, renal impairment caused by intrinsic renal disease, renal transplantation, urinary tract modifications, pyelonephritis and normal urinary tract anatomy, and at least one of the following signs or symptoms: chills or rigours associated with fever or hypothermia, flank pain (pyelonephritis) or pelvic pain (cUTI), dysuria or urinary frequency, or urinary urgency, costovertebral angle tenderness on physical examination and either urine culture with at least $105 \mathrm{CFU} / \mathrm{mL}$ or greater of a uropathogen (no more than two species) or at least one blood culture growing possible uropathogens (no more than two species) with no other evident site of infection. These inclusion criteria are in accordance to the definition of cUTI provided in $\operatorname{ref}^{17}$. The analysis presented in this paper focuses on patients admitted to hospital because of cUTI only; we do not include patients admitted for other reasons who developed cUTI during hospitalisation. The reason is that in the case of latter it is not possible to isolate the incremental cost of cUTI without a matched control group, that is, comparing similar patients with and without cUTI during their hospital stay (see, eg, ref 18). Our data indicate that the proportion of cUTI that are the cause of hospital admission is $65 \%$ versus a $35 \%$ that develop cUTI during hospitalisation.

\section{Study data collection}

Data were collected retrospectively for all cUTI episodes at participating hospitals during the study period. For all patients, a standardised set of information was recorded. 
This consisted of demographics, comorbidities including those required to calculate a modified Charlson score, ${ }^{19}$ place of acquisition of infection, infection source and severity, microbiological data, imaging test data, infection management, antibiotic therapy, outcomes, details of discharge and readmissions. The follow-up period was 2 months after discharge from the admitting hospital.

The perspective of the cost analysis was the hospital provider, as we focus on hospitalised patients with cUTI, and this is where the majority of the cost burden falls. ${ }^{20} 21$

Study size was defined based on the primary outcome measure of the main study, that is. treatment failure rate between MDR bacteria and other pathogens. ${ }^{16}$

\section{Estimating the cost per case of cUTI}

We collected information on healthcare resource utilisation attributed to cUTI for each episode in the dataset. The healthcare components collected were: (1) length of hospital stay (LOS) (general ward and intensive care unit (ICU)), (2) diagnostic and follow-up tests, (3) urological interventions and haemodialysis, (4) antibiotic treatment before, during and after hospitalisation and (5) hospital readmissions and outpatient visits within 60 days of discharge. For each component, a comprehensive list of specific items was compiled and reviewed by a clinical expert so that it included only healthcare resources that could be attributed to cUTI.

For unit costs, we planned to use the tool developed by WHO-CHOICE health service delivery costs, ${ }^{22}$ which provides information on the unit costs of bed-days and outpatient visits across 191 countries. Unfortunately, unit costs from this tool are only available for inpatient and outpatient visits, and for 2007-2008, and therefore they could not be used in our study. Instead, unit cost data for each cost item were collected for each country by means of a questionnaire sent to the principal investigators of all participating sites. The questionnaire was provided as an online and paper version and included the list of all healthcare services identified for the management of cUTI (see supplementary material 1). The response rate for the questionnaire was $90 \%$ (18 out of $20)$. We received at least one response from each country. However, despite efforts to facilitate the complete fulfilment and harmonisation of the questionnaires, responses from some of the sites had missing values for key healthcare costs items, such as the cost of a day in hospital and for the most frequent diagnostic tests and treatment procedures. Furthermore, some sites provided the data in terms of user charges instead of the cost incurred by the hospital in the provision of the services. As a result, we observed a large degree of variation in unit costs across sites that was not attributable only to differences in actual costs between regions. Therefore, we generated a single set of unit costs based on the mean values across three sites within the same country, Spain, which provided consistently estimated values reflecting hospital costs for all the items included in the questionnaire. Using a common set of unit costs across all patients means that any observed variation in costs is due to differences in healthcare resource use. We discuss the limitations of this approach in the discussion section.

For antibiotic therapy, we estimated the cost per mg for each drug for which unit cost data were available and applied the mean cost per mg to the remaining therapies. We estimated the cost per day with antibiotic therapy based on the dosage and frequency recorded for each drug, which was then combined with the duration of the treatment to estimate total antibiotic therapy costs. Patients might receive more than one antibiotic drug at the same time; in that case, they count as separate antibiotic therapy days. Patients with total hospital LOS >200 days were excluded (three observations) as these were deemed to be due to coding errors.

We computed means and SD as well as medians and IQRs for the cost per case, and we quantified the contribution of each cost item and overall healthcare component to the total cost per case. We also present variations in the overall cost per case by country and for different cost components. All costs were reported in 2016 euros.

Costs were calculated for each case of cUTI requiring a hospital admission. If a patient required a second hospital admission, then if this occurred within 60 days of discharge of the first admission, it was counted as a readmission and included in the cost of the first admission. If another admission occurred after 60 days postdischarge (either of the index admission or a readmission), then this was counted as a separate case (observation) in the data.

\section{Factors associated with cUTI-related healthcare costs}

The analysis of the factors associated with cUTI-related healthcare costs was undertaken using multivariate regression analysis using patient level cost data. The dependent variable was total cost per patient estimated as described above.

The explanatory variables were demographic factors (age and gender), comorbidities measured by the Charlson morbidity index, ${ }^{18}$ admission characteristics (urgent vs elective; and admitted from home vs from another facility), infection severity (defined as septic shock or severe sepsis), MDR profile (defined as non-susceptibility to at least one agent in three or more antimicrobial categories $^{23}$ ), episode number and 30-day mortality. We categorised the source of infection using the following definitions: (1) UTI related to indwelling urinary catheterisation including long-term, short-term or intermittent catheterisation; (2) pyelonephritis, consisting of inflammation of the kidney tissue caused by bacterial infection in patients that have no other urinary tract modification; and (3) other sources, which includes UTI related to anatomical urinary tract modification, UTI related to obstructive uropathy and UTI related to other events that do not fulfil any other category. We ran three sets of models: (1) univariate regression models for each variable separately, (2) a multivariate model including all the covariates and (3) a reduced multivariate model including only significant variables (where in 
the case of categorical variables, at least one indicator was non-significant).

Analyses were undertaken using Stata V.12. More details about the statistical methods used in the analyses are reported in the online supplementary material 2.

\section{RESULTS}

\section{Study population characteristics}

Data were collected on 653 cUTI episodes in 637 patients (mean number of episodes per patient: 1.04). There were missing data on LOS for nine episodes, so mean costs per case were computed for 644 cases. Most common causative pathogens in this sample were E. coli $(58 \%)$, Klebsiella sp. (14\%), Proteus mirabilis (7\%), Pseudomonas aeruginosa $(6 \%)$ and Enterococcus sp. (5\%). This is consistent with previous studies that have found $E$. coli to be the most commonly isolated organism, especially in cUTI acquired at the community, ${ }^{24}$ which were the majority in our sample $(69 \%$ vs $31 \%$ associated to healthcare facilities).
Fifty-seven per cent of the cohort were females, and the mean age was 65.7years (table 1). Mean Charlson comorbidity score was 2.4 . Ninety-one per cent of admissions were urgent (as opposed to elective), and $85 \%$ of the patients were admitted from home (as opposed from another facility). The infection source was indwelling urinary catheterisation in $20 \%$ of cases, pyelonephritis in $27 \%$ of cases and other sources (including anatomical urinary tract modification and obstructive uropathy) in the remaining $53 \%$. Twenty-six per cent of the episodes were caused by MDR bacteria. The severity of the infection was categorised as severe sepsis or septic shock in $16 \%$ of cases. Five per cent of the sample died within 30 days of discharge. The proportion of cases collected by each country ranged from $5 \%$ in Bulgaria to $26 \%$ in Israel.

\section{Estimating the cost per case of cUTI}

Table 2 presents unit costs, resource use and total costs separately for each healthcare item as well as for each set of overall cost components. The mean (median) length of

Table 1 Summary statistics of cohort characteristics and regression analysis results of total cost per cUTI episode

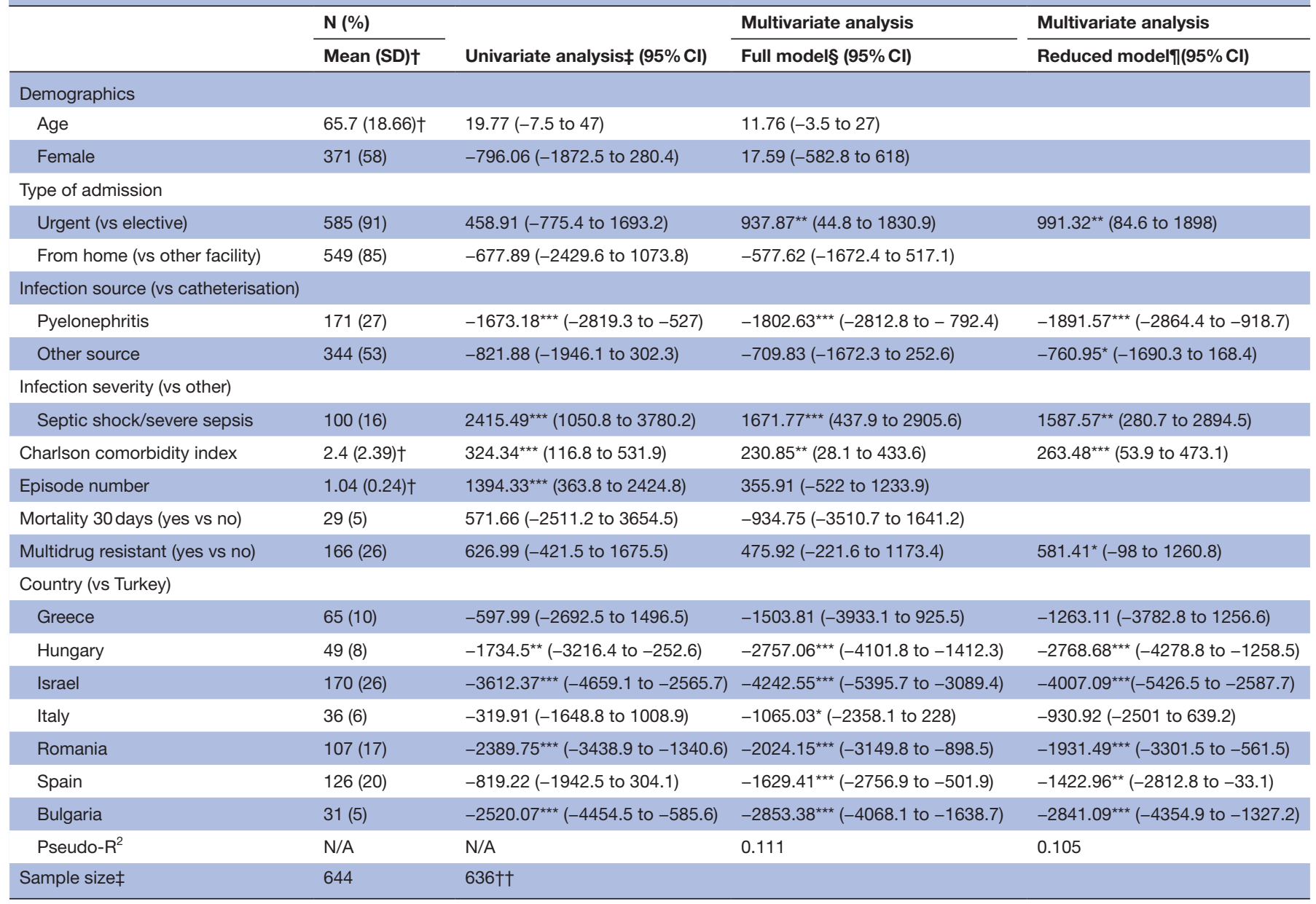

${ }^{*} \mathrm{P}<0.1$. ${ }^{\star *} \mathrm{P}<0.05 .{ }^{* \star *} \mathrm{P}<0.01$.

†Summary statistics for continuous variables are shown as mean and SDs; for categorical variables we present total number of observations and percentage.

¥Marginal effects of univariate regression models for each variable separately.

§Marginal effects of a multivariate model including all the covariates.

१Marginal effect of a reduced multivariate model including only significant variables.

††There are eight cases with missing data on mortality at 30 days. Therefore, the sample used in the regression analyses includes 636 cases out of the 644 cases for whom data on cost per case was available.

cUTI, complicated urinary tract infection; N/A, not applicable. 
Table 2 Cost per case by cost component-all countries combined

\begin{tabular}{|c|c|c|c|c|c|c|}
\hline & \multirow{2}{*}{$\begin{array}{l}\text { Unit cost } \\
(€)\end{array}$} & \multicolumn{2}{|c|}{ Resource use (units) } & \multicolumn{2}{|l|}{ Total cost $(€)$} & \multirow[b]{2}{*}{$\%$} \\
\hline & & Mean (SD) & Median [Q1-Q3] & Mean (SD) & Median [Q1-Q3] & \\
\hline \multicolumn{7}{|l|}{ Length of stay } \\
\hline General ward (days) & 477.4 & $9.25(8.49)$ & $7[5-11]$ & $4418.5(4052.4)$ & 3342 [2387-5252] & 77.4 \\
\hline \multirow[t]{2}{*}{ ICU (days) } & 1589.6 & $0.05(1.19)$ & $0[0-0]$ & $83.9(1895.4)$ & $0[0-0]$ & 1.5 \\
\hline & & $9.30(8.51)$ & $7[5-11]$ & 4502.4 (4389.9) & 3342 [2387-5252] & 78.9 \\
\hline \multicolumn{7}{|l|}{ Diagnostic tests } \\
\hline Urine culture test & 15.1 & $1.51(0.82)$ & $1[1-2]$ & $22.8(12.5)$ & 15 [15-30] & 0.4 \\
\hline Dipstick analysis & 2.8 & $0.49(0.85)$ & $0[0-1]$ & $1.3(2.3)$ & $0[0-3]$ & 0.0 \\
\hline Urinary sediment analysis & 2.6 & $1.02(0.89)$ & $1[0-1]$ & $2.6(2.3)$ & $3[0-3]$ & 0.0 \\
\hline Gram stain test & 6.3 & $0.37(0.68)$ & $0[0-1]$ & $2.3(4.2)$ & $0[0-6]$ & 0.0 \\
\hline Blood culture & 36.7 & $1.43(1.56)$ & $1[0-2]$ & $52.5(57.1)$ & 37 [0-73] & 0.9 \\
\hline Abdominal ultrasonography & 48.9 & $0.71(0.64)$ & $1[0-1]$ & 34.5 (31.3) & 49 [0-49] & 0.6 \\
\hline CT scan & 156.0 & $0.2(0.46)$ & $0[0-0]$ & $32(72.5)$ & $0[0-0]$ & 0.6 \\
\hline Pyelography & 105.1 & $0.02(0.14)$ & $0[0-0]$ & $2(14.2)$ & $0[0-0]$ & 0.0 \\
\hline \multirow[t]{2}{*}{ MRI scan } & 191.6 & $0(0.07)$ & $0[0-0]$ & $0.9(13.1)$ & $0[0-0]$ & 0.0 \\
\hline & & & & $151(109)$ & 115 [75-201] & 2.6 \\
\hline \multicolumn{7}{|l|}{ Treatment procedures } \\
\hline Insertion of catheter & 50.0 & $0.36(0.48)$ & $0[0-1]$ & $17.8(24)$ & $0[0-50]$ & 0.3 \\
\hline Replacement of catheter & 50.0 & $0.13(0.38)$ & $0[0-0]$ & $6.5(19)$ & $0[0-0]$ & 0.1 \\
\hline Percutaneous nephrostomy & 717.6 & $0.05(0.26)$ & $0[0-0]$ & $37.9(183.8)$ & $0[0-0]$ & 0.7 \\
\hline Insertion of JJ-stent & 907.0 & $0.05(0.21)$ & $0[0-0]$ & $40.8(188.2)$ & $0[0-0]$ & 0.7 \\
\hline Abscess drainage & 557.6 & $0.01(0.12)$ & $0[0-0]$ & $6.9(69.2)$ & $0[0-0]$ & 0.1 \\
\hline Nephrectomy & 3174.0 & $0.01(0.08)$ & $0[0-0]$ & $19.7(249.6)$ & $0[0-0]$ & 0.3 \\
\hline Mechanical ventilation (days) & 350.0 & $0.12(0.99)$ & $0[0-0]$ & $41.8(346.4)$ & $0[0-0]$ & 0.7 \\
\hline \multirow[t]{2}{*}{ Renal replacement (days) } & 254.7 & $0.16(1.53)$ & $0[0-0]$ & $41.9(389.8)$ & $0[0-0]$ & 0.7 \\
\hline & & & & $213.4(764.8)$ & $0[0-50]$ & 3.7 \\
\hline \multicolumn{7}{|l|}{ Antibiotic therapy } \\
\hline Before hospitalisation (days) & 1.9 & $1.95(5.84)$ & $0[0-0]$ & $2.6(20.6)$ & $0[0-0]$ & 0.0 \\
\hline During hospitalisation (days) & 12.3 & $12.25(61.37)$ & $7[4-11]$ & $197.8(474.8)$ & 19 [7-149] & 3.5 \\
\hline \multirow[t]{2}{*}{ At discharge (days) } & 5.9 & $6(13.61)$ & $4.5[0-8]$ & $24.1(117.5)$ & $2[0-10]$ & 0.4 \\
\hline & & $1.08(4.53)$ & $0[0-0]$ & $224.6(490.3)$ & 32 [14-199] & 3.9 \\
\hline \multicolumn{7}{|l|}{ After discharge } \\
\hline Readmission (days) & 477.4 & $1.08(4.53)$ & $0[0-0]$ & $515.2(2163.4)$ & $0[0-0]$ & 9.0 \\
\hline \multirow[t]{2}{*}{ Outpatients (visits) } & 122.3 & $0.81(1.62)$ & $0[0-1]$ & $99.2(197.8)$ & 0 [0-122] & 1.7 \\
\hline & & & & $614.4(2197.5)$ & $0[0-245]$ & 10.8 \\
\hline \multicolumn{4}{|l|}{ Total (first hospital admission only) } & $5064(4847)$ & 3627 [2531-5985] & 88.8 \\
\hline \multicolumn{4}{|c|}{ Total (first hospital admission+antibiotic treatment before and after discharge) } & $5091(4844)$ & $3651[2542-6004]$ & 89.2 \\
\hline \multicolumn{4}{|c|}{$\begin{array}{l}\text { Total (hospital admission+antibiotic treatment before and after } \\
\text { discharge+readmissions and outpatients visits) }\end{array}$} & $5705(5438)$ & 3919 [2664-6655] & 100 \\
\hline
\end{tabular}

*Unit costs estimated from the three Spanish sites.

ICU, intensive care unit.

stay in hospital was 9 (7) days, and a small proportion of the total stay was in the ICU. Most patients had urine cultures, urinary sediment analyses and blood cultures undertaken, while imaging tests were rarely performed. The urological intervention most often performed was the insertion of an indwelling bladder-catheter. The mean number of antibiotic therapy days before, during and after hospitalisation were 2, 12 and 6 days, respectively. Nearly $10 \%$ of patients 
Table 3 Mean cost per case by cost component-by country

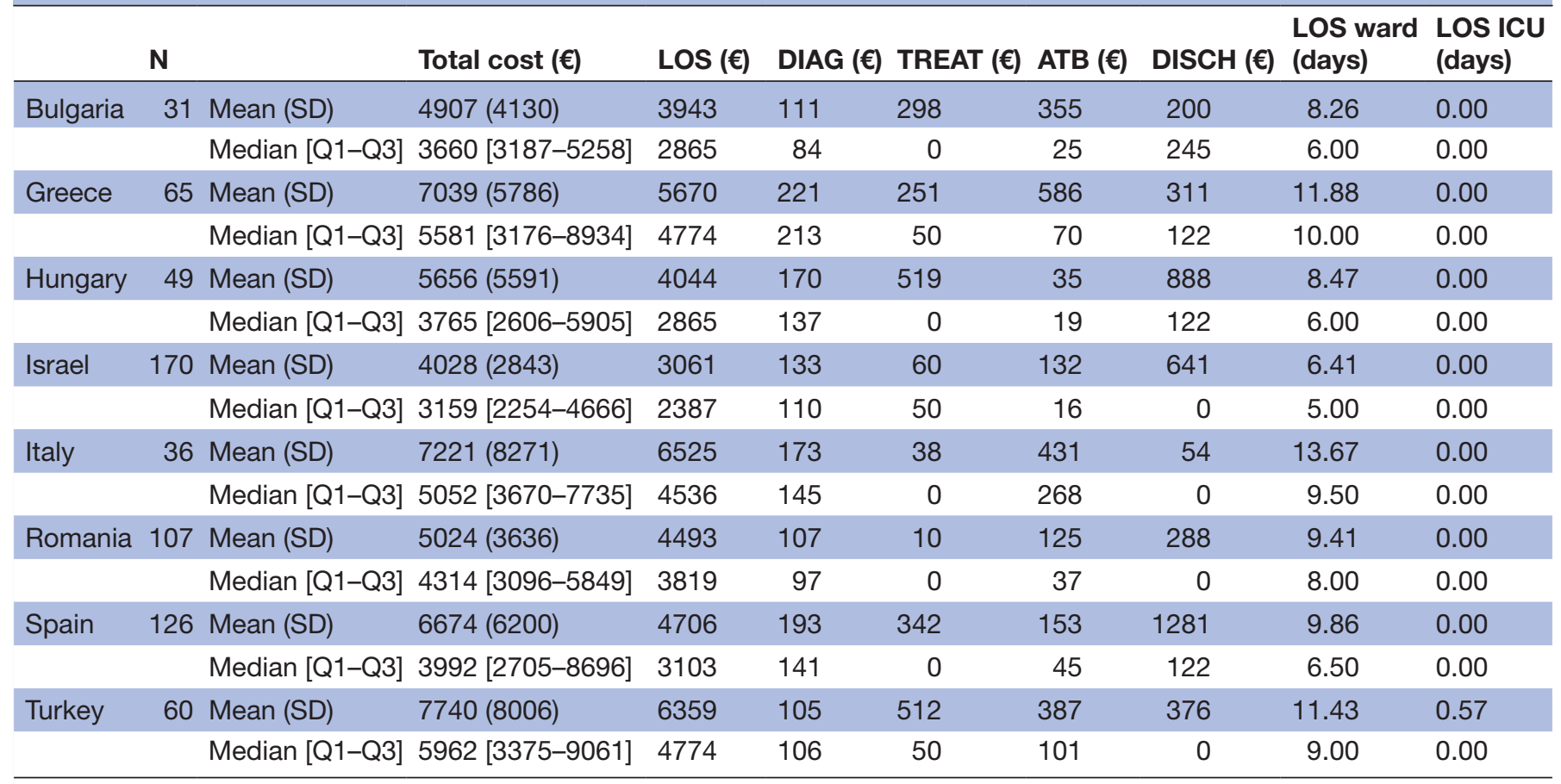

${ }^{*}$ Holding unit costs constant.

ATB, antibiotic therapy; DIAG, diagnostic test; DISCH, after discharge (readmission and outpatient visits); ICU, intensive care unit; LOS, length of stay; TREAT, treatment procedures.

were readmitted to hospital due to a cUTI recurrence, with a mean readmission stay across the full sample of 1 day (11 days among the subsample of readmitted patients). The mean number of outpatient visits per patient within 60 days of hospital discharge was 0.8 .

The mean (median) costs per case were: (1) including costs incurred during the first hospital admission: $€ 5064$ (€3627); (2) plus antibiotic therapy before and after discharge: $€ 5091$ (€3651); and (3) plus outpatient visits and hospital readmissions within 60 days of discharge: $€ 5705$ (€3919).

The cost per case was largely driven by the cost due to the length of stay in hospital, which accounted for nearly $80 \%$ of the total cost. This was followed by the contribution of the cost of readmissions and outpatient visits after discharge $(11 \%)$, treatment procedures $(4 \%)$, antibiotic therapy $(4 \%)$ and diagnostic tests $(3 \%)$.

There was variation in the mean cost per cUTI case by country, with a largest mean (median) value of $€ 7740$ (€5962) in Turkey and a lowest value of $€ 4028$ $(€ 3159)$ in Israel (table 3$)$. Note that variations in total costs shown in this table are only due to variations in the management of patients with cUTI, including LOS, as unit costs of healthcare services are held constant across all countries. Table 3 also shows variations by cost components between countries. This suggests that differences in LOS are the main reason of the observed differences in total costs between countries; the mean stay in hospital in a general ward varies from 6 days in Israel to 14 days in Italy.

\section{Factors associated with cUTI-related healthcare costs}

The statistically significant drivers of cUTI-related healthcare costs were (table 1): type of admission (with urgent admissions exhibiting a higher cost than elective admissions); source of infection (with catheterisation associated to higher costs compared with other sources); the infection severity (septic shock and severe sepsis showing a larger cost); the Charlson comorbidity index (with larger values associated to a higher cost); MDR profile (episodes presenting MDR showing a higher cost; only significant at $10 \%$ significance level); and country (with most countries exhibiting a significant lower cost than Turkey).

\section{DISCUSSION}

In this study we have measured the cost per episode of patients hospitalised due to cUTI in eight countries with high prevalence of MDR and explored the factors that explained variations in cUTI-related healthcare costs. The mean cost per hospitalised cUTI case in our data was estimated as $€ 5700$, corresponding to the costs of a hospital stay of 9 days on average and including the costs of specific diagnostic and treatment procedures, as well as antibiotic therapy, readmissions due to cUTI reoccurrence and outpatient visits after discharge. As expected, the largest cost component was LOS, but it is also worth noting that the cost of antibiotic treatment exceeded that incurred to perform diagnostics tests, and it was also larger than the costs due to any other treatment received by these patients. The cost per case varied across 
countries, mainly due to differences in LOS in hospital among patients with cUTI. These differences in LOS do not appear to be related to the models of healthcare in each participating country-the countries with longest LOS, Turkey, Italy and Greece, have different healthcare systems, that is, social insurance system, national health system and mixed system, respectively. Several factors might explain these cross-country variations, including financial incentives inherent in hospital payments methods, availability of beds and the expansion of early discharge programmes that allow patients to return to their homes to receive follow-up care. ${ }^{25}$

Over and above differences across countries, our analysis also identifies a series of factors associated with higher cUTI-related healthcare costs. Urgent admissions, for infections due to an indwelling urinary catheterisation, resulting in septic shock or severe sepsis, in patients with a higher comorbidity index and presenting MDR were related to a higher cost. The presence of catheter on admission and the Charlson comorbidity index have also been found in the literature to increase costs of adult patients hospitalised with UTI, together with time to appropriate therapy. ${ }^{13}$ Another study found males, patients with chronic renal failure, ESBL production and outpatient parenteral antibiotic therapy to be associated with higher costs in patients with UTI admitted to hospital. $^{15}$

Our cost estimates are in line with previous studies that have focused on similar patient groups. Esteve-Palau et $a l^{15}$ estimated a mean cost per patient hospitalised with symptomatic UTI caused by ESBL-producing $E$. coli of $€ 4980$ in one hospital in Spain, excluding readmissions. The cost was significantly lower, $€ 2612$, among patients with UTI due to non ESBL-producing E. coli. Cardwell et $a l^{13}$ analysed data on adults patients with a discharged diagnosis code for UTI in one hospital in the USA and found a mean hospitalisation cost of $\$ 7586$. The costs of nosocomial UTI infections and UTI infections seen in primary care have been shown to be lower. For instance, Saint ${ }^{12}$ estimated the incremental cost of nosocomial UTIs of $\$ 676$ and catheter-related bacteraemia of $\$ 2836$ per case. Tambyah et $a l^{11}$ reported that the mean incremental hospitalisation cost attributable to nosocomial catheter-associated UTI was \$589. However, studies that focused on UTI infections treated in primary care have reported a mean cost between $€ 70^{9}$ and $€ 236^{10}$ per episode.

This is the first study to examine costs of hospitalised patients due to cUTI from a multinational point of view. Moreover, it is focused on countries with a high prevalence of MDR bacteria where cUTI impose a significant burden. In addition, the study estimated the mean cost per case from a bottom-up perspective, which provided a high level of granularity and the basis for the assessment of sources of variation and drivers of healthcare costs. However, the study also has a number of limitations. The design of the study did not include a control group to assess the extra length of stay and excess costs of patients who are admitted to hospital due to a different condition and develop UTI during their hospitalisation. Therefore, we focused in this paper on the analysis of patients who are admitted because of a cUTI. This is to avoid the overestimation that would result among cases admitted for other reasons for whom we cannot isolate the incremental costs that are due to cUTI only. A second limitation of the analysis is that, as discussed in the Methods section, country-specific unit cost data were not appropriate for most countries, and therefore we applied the same set of unit costs, as estimated in one country, Spain, to the rest of the countries. While this approach allowed us to explore variations in healthcare costs that are due to differences in the management of patients with cUTI across countries rather than due to differences in the unit costs of services, it limits the validity of the country-specific estimates. To further explore the heterogeneity of country-specific estimates, we planned to use the tool developed by WHO-CHOICE health service delivery costs, ${ }^{22}$ which provides information on the unit costs of bed-days and outpatient visits across 191 countries. The information from this dataset indicates that variations in cost estimates across countries would be enhanced if country-specific unit costs were used. The countries with the highest unit costs according to this tool, that is, Spain, Italy and Greece, are among the countries with higher episode costs based on healthcare utilisation in our analysis, while the country with the lowest unit cost, Bulgaria, has an estimated episode cost among the lowest in this study. Unfortunately, unit costs values from this tool are only available for inpatient and outpatient visits, and for 2007-2008, and therefore they could not be used to construct country-specific estimates. In addition, we acknowledge that the theoretical proper unit cost for a resource is its opportunity cost (the value of the foregone benefits because the resources are not available for their next best alternative use). We take, as most previous studies, a pragmatic approach of using market prices and accounting costs. However, it is worth noting that, especially for inpatient day cost, these values might overestimate their opportunity costs. This is because most hospital costs are fixed and cannot be recouped even if the admission is avoided. ${ }^{26}$ We also acknowledge that the number of observations included in the study for some countries is low, ranging from 31 to 170 , which might restrict the generalisability of country-specific findings. The explanatory power of our models was also found to be low, which might suggest that there are other factors not captured by the observed variables included in our models that explain variation in healthcare costs, such as hospital policy on LOS. Finally, the perspective of the analysis was that of the hospital provider; however, if a societal perspective had been considered wider costs related to cUTI should had been taken into account, such as patients' costs and productivity losses due to illness, as well as cost incurred by primary care settings, including these costs would increase the costs of cUTI.

In conclusion, this study showed the costs of patients hospitalised due to cUTI are substantial but identified wide differences between countries, especially due to differences in length of stay in the hospital. These findings suggest that a 
better knowledge of the reasons for longer length of stays in some countries could facilitate a better standardised quality of care for patients with cUTI and to allow a more efficient allocation of healthcare resources. The factors associated with higher cUTI-related healthcare costs identified by this study also shed light onto some implications for policy and planning. Prompting preventive measures to minimise cost of hospitalisation might be aimed at increasing the population's knowledge of symptoms and signs of infection, in order to encourage patients to attend primary care facilities earlier, especially those with comorbidities or indwelling urinary catheters, and thus to avoid the development of severe forms of illness after the onset of symptoms and avoid the need for urgent admissions.

\section{Author affiliations}

${ }^{1}$ UCL Department of Applied Health Research, University College London, London, UK

${ }^{2}$ Departamento de Métodos Cuantitativos en Economía y Gestión, Universidad de Las Palmas de Gran Canaria, Las Palmas, Spain

${ }^{3}$ Infectious diseases Department, Hospital Universitari de Bellvitge-IDIBELL,

Barcelona, Spain

${ }^{4}$ Spanish Network for the Research in Infectious Diseases (REIPI RD12/0015), Instituto de Salud Carlos III, Madrid, Spain

${ }^{5}$ AiCuris Anti-infective Cures GmbH, Wuppertal, Germany

${ }^{6}$ Informatics Unit, Fundació Institut Català de Farmacologia, Barcelona, Spain

${ }^{7}$ Department of Medical Microbiology, Southmead Hospital, North Bristol NHS Trust, Bristol, UK

${ }^{8}$ Julius Center for Health Sciences and Primary Care, University Medical Center

Utrecht, Utrecht, The Netherlands

${ }^{9}$ Department of Medicine E, Beilinson Hospital, Rabin Medical Center, Petah Tikva, Israel

${ }^{10}$ Sackler Faculty of Medicine, Tel-Aviv University, Petah Tikva, Israel

Acknowledgements We would like to thank the RESCUING Study Group and study sites' principal investigators.

Collaborators RESCUING Study Group and Study Sites principal investigators: Dora Tancheva, Rossitza Vatcheva-Dobrevska, Sotirios Tsiodras, Emmanuel Roilides, Istvan Várkonyi, Judit Bodnár, Aniko Farkas, Mical Paul, Yehuda Carmeli, Emanuele Durante Mangoni, Cristina Mussini, Nicola Petrosillo, Andrei Vata, Adriana Hristea, Julia Origüen, Jesus Rodriguez-Baño, Arzu Yetkin and Nese Saltoglu.

Contributors Conceptualisation: all authors; acquisition of data: all authors; analysis of data: LVT, MP, ES, LL and SM; writing - original draft preparation: LVT, MP, ES, LL and SM; writing - review and editing: all authors; agree with manuscript results and conclusions: all authors.

Funding This research project receives support from the Innovative Medicines Initiative Joint Undertaking under grant agreement $n^{\circ} 115523,115620,115737$ resources of which are composed of financial contribution from the European Union Seventh Framework Programme (FP7/2007-2013) and EFPIA companies in kind contribution. The research leading to these results was conducted as part of the COMBACTE-MAGNET consortium. For further information please refer to www. COMBACTE.com

Competing interests Within the IMI project, AiCuris provided support for the institutions of the following Researchers: LVT, MP, ES, JMV, MS, SG, JG, NC, LH, NE-R, JC, AM, TB, LL and SM. IW, ChV, CuV and IA are employees of AiCuris Antiinfective Cures GmbH, an EFPIA (European Federation of Pharmaceutical Industries and Association) member in the IMI JU. Costs related to the research contribution by IW, ChV, CuV and IA are borne by AiCuris Anti-infective Cures GmbH and considered in-kind contribution under the IMI JU scheme.

Patient consent Not required.

Ethics approval Local Ethics Committees of Research in each participating site.

Provenance and peer review Not commissioned; externally peer reviewed.

Data sharing statement № additional data available.
Open Access This is an Open Access article distributed in accordance with the Creative Commons Attribution Non Commercial (CC BY-NC 4.0) license, which permits others to distribute, remix, adapt, build upon this work non-commercially, and license their derivative works on different terms, provided the original work is properly cited and the use is non-commercial. See: http://creativecommons.org/ licenses/by-nc/4.0/

(C) Article author(s) (or their employer(s) unless otherwise stated in the text of the article) 2018. All rights reserved. No commercial use is permitted unless otherwise expressly granted.

\section{REFERENCES}

1. Lichtenberger P, Hooton TM. Complicated urinary tract infections. Curr Infect Dis Rep 2008;10:499-504.

2. Nicolle LE. A practical guide to the management of complicated urinary tract infection. Drugs 1997;53:583-92.

3. Levison ME, Kaye D. Treatment of complicated urinary tract infections with an emphasis on drug-resistant gram-negative uropathogens. Curr Infect Dis Rep 2013;15:109-15.

4. Foxman B. Epidemiology of urinary tract infections: incidence, morbidity, and economic costs. Am J Med 2002;113(Suppl 1A):5S-13.

5. European Centre for Disease prevention and Control. Point prevalence survey of healthcare-associated infections and antimicrobial use in European acute care hospitals. Stockholm: European Centre for Disease prevention and Control, 2013.

6. Cassini A, Plachouras D, Eckmanns T, et al. Burden of Six Healthcare-Associated Infections on European Population Health: Estimating Incidence-Based Disability-Adjusted Life Years through a Population Prevalence-Based Modelling Study. PLoS Med 2016;13:e1002150.

7. Rosenberg M. Pharmacoeconomics of treating uncomplicated urinary tract infections. Int J Antimicrob Agents 1999;11:247-51.

8. Foxman B, Barlow R, D'Arcy $\mathrm{H}$, et al. Urinary tract infection: self-reported incidence and associated costs. Ann Epidemiol 2000;10:509-15.

9. François M, Hanslik T, Dervaux B, et al. The economic burden of urinary tract infections in women visiting general practices in France: a cross-sectional survey. BMC Health Serv Res 2016;16:365.

10. Ciani O, Grassi D, Tarricone R. An Economic Perspective on Urinary Tract Infection: The "Costs of Resignation". Clin Drug Investig 2013;33:255-61.

11. Tambyah PA, Knasinski V, Maki DG. The direct costs of nosocomial catheter-associated urinary tract infection in the era of managed care. Infect Control Hosp Epidemiol 2002;23:27-31.

12. Saint S. Clinical and economic consequences of nosocomial catheter-related bacteriuria. Am J Infect Control 2000;28:68-75.

13. Cardwell SM, Crandon JL, Nicolau DP, et al. Epidemiology and economics of adult patients hospitalized with urinary tract infections. Hosp Pract 2016;44:33-40.

14. MacVane SH, Tuttle LO, Nicolau DP. Impact of extended-spectrum $\beta$-lactamase-producing organisms on clinical and economic outcomes in patients with urinary tract infection. $J$ Hosp Med 2014;9:232-8.

15. Esteve-Palau E, Solande G, Sánchez F, et al. Clinical and economic impact of urinary tract infections caused by ESBL-producing Escherichia coli requiring hospitalization: A matched cohort study. $J$ Infect 2015;71:667-74.

16. Shaw E, Addy I, Stoddart M, et al. Retrospective observational study to assess the clinical management and outcomes of hospitalised patients with complicated urinary tract infection in countries with high prevalence of multidrug resistant Gram-negative bacteria (RESCUING). BMJ Open 2016;6:e011500.

17. U.S. Department of Health and Human Services Food and Drug Administration. Complicated Urinary Tract Infections: Developing Drugs for Treatment Guidance for Industry, 2015. https://www.fda. gov/downloads/Drugs/./Guidances/ucm070981.pdf.

18. Roberts RR, Scott RD, Hota B, et al. Costs attributable to healthcareacquired infection in hospitalized adults and a comparison of economic methods. Med Care 2010;48:1026-35.

19. Charlson ME, Pompei P, Ales KL, et al. A new method of classifying prognostic comorbidity in longitudinal studies: development and validation. J Chronic Dis 1987;40:373-83.

20. Turner RM, Wu B, Lawrence K, et al. Assessment of Outpatient and Inpatient Antibiotic Treatment Patterns and Health Care Costs of Patients with Complicated Urinary Tract Infections. Clin Ther 2015;37:2037-47. 
21. Bader MS, Hawboldt J, Brooks A. Management of complicated urinary tract infections in the era of antimicrobial resistance. Postgrad Med 2010;122:7-15.

22. World Health Organization. WHO-Choice unit cost estimates for service delivery. $2011 \mathrm{http} / / / \mathrm{www}$.who.int/choice/country/WHOCHOICEunit_cost_estimates_2007_2008.xls.

23. Ahmed NH, Hussain T, Biswal I. Comparison of etiological agents and resistance patterns of the pathogens causing community acquired and hospital acquired urinary tract infections. J Glob Infect Dis 2014:6:135-6.
24. Magiorakos A-P, Srinivasan A, Carey RB, et al. Multidrug-resistant, extensively drug-resistant and pandrug-resistant bacteria: an international expert proposal for interim standard definitions for acquired resistance. Clinical Microbiology and Infection 2012;18:268-81.

25. OECD. Health at a Glance 2017: OECD Indicators. Paris: OECD Publishing, 2017.

26. Stewardson AJ, Harbarth S, Graves N. Valuation of hospital beddays released by infection control programs: a comparison of methods. Infect Control Hosp Epidemiol 2014;35:1294-7. 